\title{
Current Depression Interventions for Older Adults: A Review of Service Delivery Approaches in Primary Care, Home-Based, and Community-Based Settings
}

\author{
Duy Nguyen • Catherine M. Vu
}

Published online: 9 January 2013

(C) Springer Science+Business Media New York 2013

\begin{abstract}
Although evidence-based depression interventions exist, relatively few older adults seek care from mental health specialists. Moreover, studies that examine the models used to deliver mental health services have been limited. In light of an aging and diversifying population, this article reviews current depression interventions for older adults in non-mental health clinics. A total of 21 articles were included in this review focusing on depression interventions conducted in primary care, home-based, and community-based settings. Notably, care manager-based interventions and problem solving interventions reduced depression across settings. Very few studies focused on racial/ethnic minority populations. Models of geriatric service delivery are highlighted and implications for ethnic minority older adults are discussed in light of health care reform.
\end{abstract}

Keywords Mental health · Elder · Non-traditional settings · Stigma $\cdot$ Service use $\cdot$ Aging $\cdot$ Diverse populations $\cdot$ Service delivery systems

\section{Introduction}

As the population in the U.S. ages, major depression in older adults will be an increasing concern that has significant public health implications. More than $20 \%$ of older adults in hospital and institutional long term care settings experience significant depressive symptoms while 5-15\% of older adults living in the community present with signs of depression [1-4]. Left untreated, depression leads to

D. Nguyen $(\bowtie) \cdot$ C. M. Vu

Silver School of Social Work, New York University,

1 Washington Square North,

New York, NY 10003, USA

e-mail: duy.nguyen@nyu.edu significantly higher health care costs [5], increased disability, and diminished quality of life [6]; negatively impacts other health outcomes [7]; and increases risk for suicide [8] and overall mortality [9]. Despite the prevalence of mental health problems, mental health care is sought by relatively few older adults [10], particularly those from ethnic minority backgrounds [11]. Meanwhile, the mental health and substance abuse treatment workforce is ill-prepared for an aging population [12]. Studies examining the effects of race, culture, and ethnicity on mental health status and service utilization suggest that the current mental health system under-serves racial and ethnic minority groups [13] due to a biased system that misdiagnoses the individual based on racial and neighborhood characteristics [14, 15] or numerous barriers associated with service utilization including language access issues, a fragmented health care system, lack of insurance, and a lack of knowledge of existing resources $[16,17]$. Translational research that focuses on dissemination and intervention studies has the potential to improve population health [18] and mental health [19], but not enough research has been done on older adults. In light of population aging and the growing diversity among older adults, the development of effective and culturally appropriate interventions is critical in addressing the health and mental health disparities affecting minority older adults.

The purpose of this review is to examine the most current literature on interventions used to treat depression among older adults. Of particular interest are interventions targeting older adults from ethnic minority backgrounds. Currently, depression in older adults is treated mainly in primary care or home-based settings, as opposed to specialty mental health settings. These care settings do not always take into consideration the influence of the social context of service delivery on service use. A range of social and organizational factors work in concert to affect the mental health behaviors of older adults, including perceived stigma about mental 
illness and help-seeking, beliefs and attitudes toward emotional problems, awareness of problems and available services, as well as the availability of ageappropriate and culturally appropriate mental health care [12]. Stigma is a prominent issue affecting decisions to seek mental health care, but mental health service delivery models generally have not considered the New Freedom Commission's recommendations to provide services in low-stigma settings to address unmet needs $[20,21]$. The experiences of depression in late life due to age-related health and changing social relationships presents the opportunity to intervene in settings commonly used by older adults. This analysis reviews the literature on a range of care settings and explores the potential use of non-traditional mental health settings to facilitate access to depression care for ethnic minority older adults.

\section{Methods}

This review captures a broad range of mental health interventions for older adults over the age of 55 years. Using a variety of key terms (e.g. depression, mental health, older adults, interventions) and databases (e.g. PsychInfo, PubMed, Web of Knowledge), the literature search identified 104 articles published between January, 2011, and October, 2012 (See Appendix A for a complete list of search terms and sources). We then reviewed the titles and abstracts to identify serviceoriented intervention studies, and exclude studies that focused on the effectiveness of pharmacotherapy or reported on population-based surveys. This review yielded 27 articles; of these, 21 were included for analysis in this paper. Of those excluded articles, one focused on a population other than older adults, while another that described an intervention was preliminary and did not report any results. Two were conducted in mental health clinics, while another two studies focused on care for the institutionalized.

Of the articles included in this review, five presented findings from meta-analyses or systematic reviews irrespective of location $(n=5)$. The remaining depression intervention articles were categorized by setting: primary care, home-based, or communitybased. Studies were included in the primary care category when participants were identified and recruited in primary care settings $(n=6)$. Studies were considered home-based if the study intervention was delivered only in the home $(n=6)$. Studies that reported any contact with any organization or program outside the home were classified as community-based $(n=4)$.

\section{Results}

Meta-analyses and Systematic Reviews

Recently published literature syntheses and meta-analyses examining the effectiveness of depression treatment modalities span a number of service delivery settings. A review of home health-based interventions reported positive results from a series of problem solving-based intervention studies with home health professionals and patients [22]. For example, home care nurses improved their identification of depression through the Training in the Assessment of Depression program. Subsequently, the Depression CAREPATH, a depression care manager intervention targeting nurses working with home healthcare patients with depression was tested and found to improve the identification of depressive symptoms among patients, improved medication adherence and improved depression-related education. Kionsis and colleagues' [23••] thorough review of community-based approaches found that no psychosocial interventions met the criteria of having two randomized control trials conducted by independent investigators for being efficacious for the acute treatment of late-life Major Depressive Disorder. The authors identified Problem Solving Therapy (PST), Cognitive Behavioral Therapy (CBT), and the Treatment Initiation and Participation (TIP) to be of demonstrated efficacy with one RCT showing improvement on depression outcomes in relation to the control group. Each of these approaches is likely to be efficacious pending replication by additional independent investigators. The authors did not find studies of sufficient rigor that provided statistically significant support for the use of Interpersonal Psychotherapy (IPT) or supportive therapy for the treatment of depression among older adults.

Forsman and colleagues [24.•] reviewed prospective clinical trials interventions designed to prevent the onset of depression among older adults targeting psychosocial factors, rather than biological causes of depression. The authors included intervention studies targeting physical exercise, skill training, group support, reminiscence, social activities, and multicomponent interventions. Their analysis of the pooled effects of 30 studies showed a modest, but statistically significant reduction in depressive symptoms [24••]. In particular, social support prevention interventions had a small, significant effect on the reduction of depression symptoms. The authors concluded that interventions seeking to promote social activity among older adults with depression are a promising area of geriatric research and practice that warrant further investigation $[24 \cdot \bullet]$.

A small meta-analysis was performed on four studies focused on testing interventions based on behavioral principles, such as increasing access to positive reinforcement or learning about depression symptom management [25]. 
These behavioral interventions were compared to other approaches, such as treatment as usual, supportive counseling, or interventions based in CBT, IPT, or psychodynamic psychotherapy. Though no differences were found between behavioral and comparison interventions on self-rated or clinician-rated depression measures, behavioral interventions resulted in improved depression scores on the clinician-rated Hamilton Depression Rating Scale when compared to wait-list controls [25].

Rather than compare specific treatment modalities, Shearer and colleagues [26] conducted a review of a variety of empowerment interventions, which sought to understand the process and consequences of efforts by the older adults to exert control and influence over the decisions that their life, including perceptions of personal control and behaviors to realize control. Among the four reviewed studies that included depression outcomes, three studies reported significant reductions in depression scores from empowerment interventions. For example, as cited in Shearer's review [26], a test of an empowerment intervention for patients with end stage renal disease showed participants in the experimental condition showed statistically significant improvement on Beck Depression Inventory scores.

\section{Primary Care}

Much research in recent decades has focused on the delivery of depression care in medical settings. Interventions that integrate a collaborative care model with PST in primary care settings effectively treat depression [27••]. For older adults, the Improving Mood Promoting Access to Collaborative Treatment (IMPACT) and Prevention of Suicide in Primary Care Elderly: Collaborative Trial (PROSPECT) $[12,22,27 \bullet \cdot]$ interventions, which will be described further, have demonstrated effectiveness for promoting access to depression care and improved depression and health outcomes. Recent articles that focus on addressing depression in primary care settings either build upon existing evidencebased practices (e.g. PST and collaborative care) or focus on specific dimensions that are embedded within multicomponent interventions, such as clinician-level interventions and education.

Researchers have extended the reach of multicomponent, care manager-based depression interventions to address complex mental health issues and diverse populations. For example, the IMPACT study, a depression care manager intervention in primary care, improves health and mental health outcomes among older adults, while reducing health care costs $[5,28]$. Chan et al [28] re-examined data from the IMPACT study to explore the intervention's effectiveness among older adults with PTSD. Their results showed older adults in the intervention group, both with and without PTSD, reported more depression symptom reduction compared to the control group at the conclusion of the study period and at follow up. Additionally, older adults with PTSD in the intervention group reported more depression free days at 2 year follow up compared to older adults with PTSD in the control group.

The PROSPECT study was a randomized controlled trial of a depression care manager intervention designed to reduced depression symptoms and reduces suicidal ideation among older primary care patients with depression [27••]. Bao and colleagues [27••] re-examined PROSPECT data for its impact on reducing mental health disparities among older adults with depression. Overall, the clinical decision-making process tested improved patient education and promoted adherence to psychotropic medication, but racial/ethnic minorities did not benefit from the intervention to the same degree, suggesting a need to improve its cultural competence.

Other studies have tested PST interventions in the primary care population. Enguidanos and colleagues [29] reexamined data from a randomized control trial that tested a PST intervention delivered by social workers in the primary care setting. Focusing solely on the 107 older adults enrolled in the eight-session PST condition, the researchers aimed to identify the factors related to successful problem resolution. All participants created a list of problems to be the focus of treatment, which was supplemented by mutually-agreed upon problems identified by a social worker, nurse or physician. Highlighting the importance of empowering older adult patients, problems that the patients identified were more likely to be solved compared to problems identified by professionals (i.e. social workers, nurses, or physicians). Also of note, $5 \%$ of the participants had severe levels of depression, which required more than the eight sessions that were a part of the original study design. Chu and colleagues $[30 \bullet$ ] use a case study of a primary care patient to illustrate the adaptation and cultural translation of PST for Chinese older adults. They also describe in detail the extensive process involved in cultural translation. Central to this study was the use of de-stigmatizing language to engage and retain Chinese older adults in treatment.

Two other recent studies examined features that are usually incorporated in multi-component interventions. Almeida and colleagues [31 ••] focused on targeted depression education of primary care physicians. In their cluster randomized control trial, the authors provided detailed clinical feedback on 20 older adult patients to physicians for 6 months which focused on depression identification and treatment plans, in conjunction with education materials over the course of the study. In comparison to patients of physicians in the control group, who only were asked for pooled characteristics of their patients and received general study-related newsletters, patients of physicians in the intervention condition were less likely to present with a clinically significant rating on a composite of depressive symptoms 
and self-harm behavior. The findings suggest that physician education and training can foster the early identification and intervention to address depressive symptoms and prevent the further development and identification of a major depressive disorder $[31 \bullet \bullet]$. A study by van der Weele and colleagues [32] sought to extend the stepped care approach for depression treatment in primary care settings to focus on older adults over 75 years. Their preliminary findings indicated that there were no differences in terms of cost or effectiveness between the intervention and control groups. Taken together, the findings from these two studies suggest that multi-component primary-care based interventions are more successful than piecemeal interventions.

The reviewed studies highlight the relevance of mental health to primary care, particularly in older adulthood, when depression is often comorbid with other health and mental health conditions. Furthermore, the level of involvement by the interventionist, whether it be a physician or care manager, is important in the effectiveness of any intervention in primary care.

\section{Home-Based Care}

While depression-related interventions in primary care have been a focus of research for decades, the development and evaluation of depression interventions delivered in the home is relatively recent. In the United States, the focus on home healthcare is driven by the high concentration of depression among home-bound elders and by the mandate from the Center for Medicare Services and the Older Americans Act for programs to screen for depression. Screening studies report a prevalence of $13.5 \%$ for major depression, with an additional $10.8 \%$ meeting the criteria for minor depression [22]. Among older adults receiving services at home from Meals on Wheels (MOW), estimates for prevalence of depression range from $12 \%$ to nearly half of recipients [22, 33]. Interventions developed and tested to date for this population generally resemble those that have been implemented in primary care, in that they are delivered by healthcare organizations and professionals to individual older adults with depression [34••, 35].

Choi and colleagues focused on the organizational capacity of MOW providers to meet the depression needs of the service recipients through a nationwide survey targeting MOW providers in the most populous cities in each state [33]. While almost all the programs surveyed conducted health and mental health needs and social program eligibility assessments, $60 \%$ provided information/referral for mental health needs, and $41 \%$ provided comprehensive case management, and only $12 \%$ provided any mental health counseling. The authors conclude that the ability of MOW providers to intervene depends on organizational and funding factors that vary from one state to another. While
MOW can reach underserved groups, the high degree of variability in accrediting and funding for mental health care across states increase the challenges of innovating mental health services to new settings.

Other studies have focused on in-home healthcare providers. Delaney and colleagues present the findings of an evaluation of the TRIAD program, a "train-the-trainer" model delivered to nurses and social workers to improve depression screening and intervention among home healthcare workers $[34 \cdot \bullet]$. The pre-test/post-test design for the evaluation provides initial support for the conclusion that a "train-the-trainers" model can work to facilitate the translation of interventions to the home health setting.

Three recent studies report preliminary findings for depression interventions targeting home-bound elders. An IPT-based intervention delivered by Master's level social work students reduced depression symptoms, but no significant improvement were seen in cognitive function [36]. Preliminary findings on the PATH intervention describe, through case studies, how PST that targets home healthcare recipients and their caregivers can reduce depression and improve cognitive functioning [37]. A Turkish intervention featured home visits by nursing students to deliver health and mental health care to reach underserved and sociallyisolated older adults with low cognitive functioning. These visits resulted in improved cognitive functioning, reduced depression symptoms, and decreased levels of anxiety [35].

Travers and Bartlett [38] present the results of the Silver Memories program, delivered in Australia, designed to tap into nostalgia and reminiscing in older adulthood to improve quality of life, and reduce depression symptoms and social isolation. The evaluation found the program to be highly acceptable to participants. While preliminary, the evaluation shows that a targeted intervention, broadly disseminated, can reach underserved older adults.

The home setting is a burgeoning area for targeted depression interventions. The preliminary evidence suggests that it may be possible to deliver effective care in the home setting, but additional research and more rigorous methodologies need to be employed.

\section{Community-Based Care}

While interventions in the primary care and homecare settings define and treat depression as a medical illness, recently published studies of community-based depression interventions target the relationship between daily, real world functioning and depressive symptoms. Novel interventions in community-based settings have focused on promoting physical activity and addressing psychosocial functioning.

Numerous community-based physical activity interventions also have mental health benefits. Fit and Strong and 
other similar interventions that are often delivered in senior centers have the dual benefit of promoting physical and mental health [39]. The review identified two studies of promising community-based physical activity interventions. In one, results from a 12-week skiing intervention demonstrated significant improvements in social functioning among friends and family [40]; however, it did not significantly reduce general depression. Matthews et al. [41] analyzed post hoc data from the Lifestyles Interventions and Independence for Elders Pilot, a senior center-based randomized control trial comparing the effects of a physical activity intervention with a successful aging control. Activity scores for intervention recipients with low symptoms of depression improved significantly more than scores for the successful aging control group at both the 6-month $(\mathrm{p}=.004)$ and 12month $(\mathrm{p}<.0001)$ time periods. No significant differences in activity scores were found between the seniors with high depression symptoms and the successful aging controls. However, clinically significant changes in CES-D scores were found across all three groups. Though these changes were not statistically significant, the trend suggests that physical activity has moodrelated benefits for older adults in general, including those with high levels of depression symptoms [41].

Two recent research studies delivered psychosocial interventions to address depression. One was the Wellbeing Project [42], a group CBT-based intervention to improve psychosocial functioning among older adults in England who were not otherwise receiving mental health services. This six-session psycho-education series, delivered in weekly sessions by a psychologist, targets older adults (over the age of 55 years) with mild to moderate limitations due to mental health problems. Preliminary findings for the intervention were promising: psychological functioning improved in six target domains, and these improvements remained at 4 week follow-up. The other study was a randomized clinical trial of an intervention entitled Beat the Blues, $[43 \cdot \bullet$ ], an innovative community-based program that reaches older African Americans. This multi-component intervention extends existing depression interventions in the senior center setting by featuring components that are culturally relevant for older African Americans. The results of this rigorous study showed that the cost-benefit ratio for delivering the program in a senior center is favorable compared with the use of psychotropic medication.

Interventions in community-based settings address depression through a direct focus on improved psychosocial function, or indirectly by promoting physical activity. The studies in this review suggest that psychosocial interventions targeting depression in community-based settings can be effective.

\section{Discussion}

This analysis reviews the recent literature on depression interventions in a range of care settings. Many studies with rigorous research designs delivered depression interventions in primary care and home-based setting to facilitate access to treatment $[27 \bullet \bullet, 28,31 \bullet \bullet, 32,37]$. The studies reviewed here spanned diverse service delivery settings and treatment approaches. Notably, care manager-based interventions in all three service delivery settings (i.e. primary care, homebased, and community-based) were effective at reducing depression $[22,27 \bullet \bullet, 28,43 \bullet \bullet]$. Increasingly, interventionists are home homecare workers [22], Bachelor's or Master's levels care managers [29, 36], and nurses [34••, 35]. With regard to treatment approaches, several studies evaluated PST as a stand-alone modality $[22,27 \bullet \bullet, 29,30 \bullet \cdot$, or as a component of a care manager intervention $[30 \bullet, 36]$. Findings were mixed on the effectiveness of supportive psychotherapy in reducing depression [22, 23••, 24••]; the evidence for the effectiveness of PST was stronger, suggesting that it merits more study using more rigorous research designs $[23 \cdot \bullet]$.

The number of studies evaluating depression interventions in community-based settings lagged behind studies in primary care and home-based settings. Given that older adults are less likely to seek specialty mental health services than younger persons [44], the promising outcomes of community-based interventions should be further explored for their potential capacity to engage this population. Providing interventions in these settings will be of particular interest in light of health care reform as community-based organizations will likely become the primary gateway to service delivery through their involvement in health homes, a care management system promoted by the Patient Protection and Affordable Care Act that involves a network of organizations working together to coordinate and provide health and mental health services.

Relatively few studies in this review targeted racial/ethnic minority populations. The dearth of studies focused on racial/ ethnic minorities is concerning, given the size and growth of populations of color, and a historical lack of representation of racial/ethnic minorities in mental health intervention studies [13]. Two studies are notable for their efforts to adapt and translate established evidence-based interventions for use with older racial/ethnic minorities $[30 \bullet \bullet, 43 \bullet \bullet]$. The process to adapt and translate existing evidence-based interventions is labor intensive, but necessary to ensure that racial/ethnic minorities have equitable outcomes from evidence-based depression treatment $[27 \bullet \bullet]$. Further translational research is needed in order to extend the reach of evidence-based interventions to older, diverse populations.

As the aging population becomes increasingly diverse, there is a pressing need for culturally competent geriatric 
mental health care that addresses stigma. Although a large body of literature on mental health service use suggests that stigma plays a significant factor in seeking care, particularly among ethnic minorities and immigrants [45, 46], only one reviewed study $\left[30^{\bullet} \cdot\right.$ ] considered the role of stigma as a social and systemic barrier to mental health care for older minority adults at-risk for depression. As stigma inhibits mental health help-seeking, little is known about the relationship between the spaces used for mental health care and stigma.

Findings from this analysis suggest that more can be done to incorporate the New Freedom Commission's recommendations to extend mental health service delivery care in low-stigma settings [20]. For older adults, senior centers are low-stigma settings where high unmet depression needs have been documented [21]. Although senior centers offer a range of health and social services, few studies have applied rigorous methods in senior centers as a setting for mental health interventions. The use of senior centers as a community-based service delivery setting could provide an understanding of how to better engage older ethnic minority adults in mental health service use, while controlling mental health care costs $[43 \cdot \bullet]$.

The findings of this review have implications for geriatric mental health practice and policy. This review's findings suggest that home and communitybased models need to be promoted as a viable and effective means of mental health service delivery to older adults. Inter-organizational collaboration will be particularly critical in light of health care reform, which provides incentives for all organizations, regardless of service delivery models they use, to work together in health homes. Therefore, service delivery across all settings (i.e. primary care, home-based, and communitybased) should forge partnerships and collaborations across disciplines in order to provide comprehensive care and improve population health.

Currently, the financing and delivery of mental health services in general, and depression interventions in particular, are restricted by a range of social policies, especially at the state level [33]. For example, in New York State, mental health care can only be offered in designated spaces to ensure that the space is appropriate for the delivery of clinical services (i.e. adequate structure, privacy and confidentiality). However, as suggested by preliminary research with organizations serving older Asian Americans [47], the designation of physical space for mental health services may implicitly stigmatize the setting and individuals seeking mental health services, even in trusted community-based settings. As a result, state-level policies should be re-examined to allow organizations more flexibility to deliver mental health services in settings that can reach underserved populations.

\section{Conclusion}

As identified in this review, evidence-based interventions for depression care can be effectively applied in a range of settings, with notable implications for racial/ethnic minority populations. The passage and gradual implementation of the Patient Protection and Affordable Care Act offers an opportunity to extend the reach of evidence-based models of care with culturally and aging competent practices. The long term sustainability of innovative depression interventions for an aging, and diversifying population will be incumbent upon the availability of fiscal and human resources that ensures an aging, and culturally-competent workforce [12].

Disclosure No potential conflicts of interest relevant to this article were reported.

\section{Appendix A: Search Terms and Sources}

The following pre-determined search terms were used in varying combinations:

- depression

- mental health

- interventions

- older adults

- elder*

- non-traditional settings

- community

- stigma

- $\quad$ service use

Sources and databases:

- Social Work Abstracts

- Social Services Abstracts

- Web of Knowledge

- Google Scholar

- Proquest Social Sciences

- JSTOR

- Psych Info

- PubMed

\section{References}

Papers of particular interest, published recently, have been highlighted as:

•• Of major importance

1. Gallo JJ, Lebowitz BD. The epidemiology of common late-life mental disorders in the community: themes for the new century. Psych Serv. 1999;50:1158-66. 
2. Koenig H, Meador JG, Cohen HJ. Depression in elderly hospitalized patients with medical illness. Arch Intern Med. 1988;148:1929 36.

3. Morrow-Howell N, Proctor E, Choi S, et al. Depression in public community long-term care: implications for intervention development. J Behav Health Serv Res. 2008;35:37-51.

4. Parmelee P, Katz I, Lawton M. Depression among institutionalized aged: assessment and prevalence estimation. J Gerontol Med Sci. 1989;44:22-9.

5. Unützer J, Schoenbaum M, Katon W, et al. Healthcare costs associated with depression in medically ill fee-for-service Medicare participants. J Am Geriatr Soc. 2009;57:506-10.

6. Beekman AT, Penninx BW, Deeg DJ, et al. The impact of depression on the well-being, disability, and use of services in older adults: a longitudinal perspective. Acta Psychiatr Scand. 2002;105:20-7.

7. Evans DL, Charney DS, Lewis L, et al. Mood disorders in the medically ill: scientific review and recommendations. Biol Psychiatry. 2005;58:175-89.

8. Heisel MJ, Dubenstein PR. Suicide prevention in older adults. CPSP. 2005;12:242-59.

9. Adamson JA, Price GM, Breeze E, et al. Are older people dying of depression? Findings from the medical research council trial of the assessment and management of older people in the community. $\mathbf{J}$ Am Geriatr Soc. 2005;53:1128-32.

10. Neighbors HW, Woodward AT, Bullard KM, et al. Mental health service use among older African Americans: The National Survey of American Life. Am J Geriatr Psychiatry. 2008;16:948.

11. Nguyen D. Acculturation and perceived mental health need among older Asian immigrants. J Behav Health Sci Res. 2011;38:526-33.

12. Institute of Medicine. The mental health and substance use workforce for older adults: in whose hands? Washington: The National Academies Press; 2012.

13. Office of the Surgeon General. Mental health: culture, race, and ethnicity: a supplement to mental health: a report of the surgeon general. Rockville: Department of Health and Human Services; 2001.

14. Chow JC, Jaffee K, Snowden L. Racial/ethnic disparities in the use of mental health services in poverty areas. Am J Public Health. 2003;93:792-7.

15. Snowden LR. Bias in mental health assessment and intervention: theory and evidence. Am J Public Health. 2003;93:239-43.

16. Jang M, Lee E, Woo K. Income, language, and citizenship status: factors affecting the health care access and utilization of Chinese Americans. Heal Soc Work. 1998;23:136-45.

17. Loo C, Tong B, True R. A bitter bean: mental health status and attitudes in Chinatown. J Commun Psychol. 1989;17:283-96.

18. Trochim W, Kane C, Graham MJ. Evaluating translational research: a process marker model. Clin Transl Sci. 2011;4:153-62.

19. Proctor EK, Landsverk J, Aarons G, et al. Implementation research in mental health services: an emerging science with conceptual, methodological, and training challenges. Adm Policy Ment Health Serv Res. 2009;36:24-34.

20. New Freedom Commission on Mental Health: Achieving the promise: transforming mental health care in America. Final report. Rockville: DHHS Pub; 2003.

21. Bartels SJ. Improving the United States' system of care for older adults with mental illness: findings and recommendations for the President's new freedom commission on mental health. Am J Geriatr Psychiatry. 2003;11:486.

22. Pickett YR, Raue PJ, Bruce ML. Late-life depression in home healthcare. Aging Health. 2012;8:273-84.

23. • Kiosses DN, Leon AC, Areán PA. Psychosocial interventions for late-life major depression: evidence-based treatments, predictors of treatment outcomes, and moderators of treatment effects. Psychiatr Clin North Am. 2011;34:377-401. A thorough review of a range of interventions, including an examination of important variables to include to target late life depression.
24. •• Forsman AK, Schierenbeck I, Wahlbeck K. Psychosocial interventions for the prevention of depression in older adults: systematic review and meta-analysis. J Aging Health. 2011;23:387-416. An important review and meta-analysis of psychosocial interventions with considerations of which treatment modalities have demonstrated effectiveness for older adults.

25. Samad Z, Brealey S, Gilbody S. The effectiveness of behavioural therapy for the treatment of depression in older adults: a metaanalysis. Int J Geriat Psychiatry. 2011;26:1211-20.

26. Shearer NBC, Fleury J, Ward KA, O'Brien A. Empowerment interventions for older adults. West J Nurs Res. 2012;34:24-51.

27. • Bao Y, Alexopoulous GS, Casalino LP, et al. Collaborative depression care management and disparities in depression treatment and outcomes. Arch Gen Psychiatry. 2011;68:627-36. One of the few studies that looks at differential outcomes by race and ethnicity, and shows that minority populations may not benefit from the intervention to the same degree as non-Hispanic Whites.

28. Chan D, Fan M, Unutzer J. Long-term effectiveness of collaborative depression care in older primary care patients with and without PTSD symptoms. Int J Geriatr Psychiatry. 2011;26:758-64.

29. Enguidanos S, Kogan AC, Keefe B, et al. Patient-centered approach to building problem solving skills among older primary care patients: problems identified and resolved. J Gerontol Soc Work. 2011;54:276-91.

30. • Chu JP, Huynh L, Areán P. Cultural adaptation of evidencebased practice utilizing an iterative stakeholder process and theoretical framework: problem solving therapy for Chinese older adults. Int J Geriatr Psychiatry. 2011;27:97-106. A detailed documentation of the process to adapt and translate problem solving therapy to apply with Chinese older adults.

31. • Almeida O, Pirkis J, Kerse N, et al. A randomized trial to reduce the prevalence of depression and self-harm behavior in older primary care patients. Annals Fam Med. 2012;10:347-56. This article is an innovative approach to affect and improve the mental health of older adults.

32. van der Weele GM, de Waal MWM, van den Hout WB, et al. Effects of a stepped-care intervention programme among older subjects who screened positive for depressive symptoms in general practice: the PROMODE randomised controlled trial. Age Ageing. 2012;41:482-8.

33. Choi NG, Lee A, Goldstein M. Meals on wheels: exploring potential for andbarriers to integrating depression intervention for homebound older adults. Home Health Care Serv Q: J Commun Care. 2011;30:214-30.

34. • Delaney C, Fortinsky R, Doonan L, et al. Depression screening and interventions for older home health care patients: program design and training outcomes for a train-the-trainer model. Home Health Care Manag Pract. 2011;23:435-45. The article highlights the need to effectively identify new cases of depression before we can expect to engage older adults in treatment.

35. Karatay G, Akkuş Y. The effectiveness of a stimulation program on cognitive capacity among individuals older than 60 . West J Nurs Res. 2011;33:26-44.

36. Bransford CL, Choi S. Using interpersonal psychotherapy to reduce depression among home-bound elders: a service-learning research collaboration. Best Pract Mental Health: Int J. 2012;8:115.

37. Kiosses DN, Teri L, Velligan DI, Alexopoulos GS. A homedelivered intervention for depressed, cognitively impaired, disabled elders. Int J Geriatr Psychiatry. 2011;26:256-62.

38. Travers C, Bartlett HP. Silver memories: implementation and evaluation of a unique radio program for older people. Aging Mental Health. 2011;15:169-77.

39. Huges SL, Seymour RB, Campbell R, et al. Impact of the fit and strong intervention on older adults with osteoarthritis. Gerontologist. 2004;44:217-28. 
40. Finkenzeller T, Müller E, Würth S, Amesberger G. Does a skiing intervention influence the psycho-social characteristics of the elderly? Scand J Med Sci Sports. 2011;21:69-75.

41. Matthews MM, Hsu F, Walkup MP, et al. Depressive symptoms and physical performance in the lifestyle interventions and independence for elders pilot study. J Am Geriatr Soc. 2011;59:495-500.

42. Clark S, Jackson L. The wellbeing project: improving the psychological wellbeing of older adults. Work Older People. 2011;15:87-91.

43. •• Gitlin LN, Harris LF, McCoy M, et al. A community-integrated home based depression intervention for older African Americans: description of the Beat the Blues randomized trial and intervention costs. BMC Geriatr. 2012;12. This article is an example of a rigorously designed, culturally-competent program that reaches older African Americans in their community.
44. Mickus M, Colenda CC, Hogan AJ. Knowledge of mental health benefits and preferences for type of mental health providers among the general public. Psychiatr Serv. 2000;51:199-202.

45. Nadeem E, Lange JM, Edge D, et al. Does stigma keep poor young immigrant and U.S.-Born Black and Latina Women from seeking mental health care? Psychiatr Serv. 2007;58. doi:10.1176/ appi.ps.58.12.1547.

46. Gary FA. Stigma: barrier to mental health care among ethnic minorities. Issues Mental Health Nurs. 2005;26:979-99. doi:10.1080/ 01612840500280638.

47. Nguyen, D, Park, SY, Park SY: Asian neighorhoods and the mental health of older Asian Americans. Presented at the Gerontological Society of America, 65th Annual Scientific Meeting. San Diego, CA; November 14-18, 2012. 